\title{
SuperMeshing: A New Deep Learning Architecture for Increasing the Mesh Density of Physical Fields in Metal Forming Numerical Simulation
}

\author{
Qingfeng Xu $\mathbf{X}^{1,2}$ Zhenguo Nie ${ }^{1,2 *}$ Handing $\mathrm{Xu}^{1,2}$ Haosu Zhou ${ }^{3}$ Hamid Reza Attar ${ }^{3}$ Nan Li ${ }^{3} \quad$ Fugui Xie $^{1,2}$ Xin-Jun Li \\ ${ }^{1}$ The State Key Laboratory of Tribology, Department of Mechanical Engineering, Tsinghua University, Beijing 100084, China \\ ${ }^{2}$ Beijing Key Lab of Precision/Ultra-precision Manufacturing Equipments and Control, Tsinghua University, Beijing 100084, China \\ ${ }^{3}$ Dyson School of Design Engineering, Imperial College London Exhibition Rd, London, SW7 2AZ, UK
}

In metal forming physical field analysis, finite element method (FEM) is a crucial tool, in which the mesh-density has a significant impact on the results. High mesh density usually contributes authentic to an increase in accuracy of the simulation results but costs more computing resources. To eliminate this drawback, we propose a data-driven mesh-density boosting model named SuperMeshingNet that uses low mesh-density physical field as inputs, to acquire high-density physical field with 2D structured grids instantaneously, shortening computing time and cost automatically. Moreover, the Res-UNet architecture and attention mechanism are utilized, enhancing the performance of SuperMeshingNet. Compared with the baseline that applied the linear interpolation method, SuperMeshingNet achieves a prominent reduction in the mean squared error (MSE) and mean absolute error (MAE) on the test data. The well-trained model can successfully show an improved performance than the baseline models on the multiple scaled mesh-density, including $2 \times, 4 \times$, and $8 \times$. Enhanced by SuperMeshingNet with broaden scaling of mesh density and high precision output, FEM can be accelerated with seldom computational time and cost with little accuracy sacrificed.

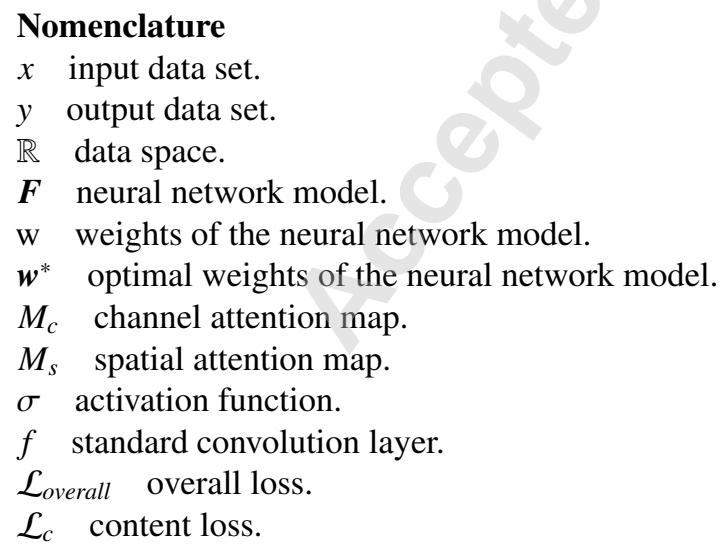

$\mathcal{L}_{p} \quad$ perceptual loss.

$\mathcal{L}_{g}$ geometric loss.

\section{Introduction}

Numerical methods, for instance, finite element method (FEM), are widely used in the engineering domain $[1,2]$ for quantitative solutions. Compared with the traditional experimental approaches, numerical methods can optimize the design and process parameters at a low cost. As shown in Fig. 1, especially the refined finite element mesh provides an essential mesh density for FEM to reach high precision [3,4].

However, to promote the accuracy of the simulation, higher mesh-density is required to be implemented during numerical simulation, which escalates the requirement of calculation resources, leading to the increase in computing time. Therefore, it is critical for FEM to generate the results that have high mesh-density while scaling down time consumption.

The prediction tasks for physical field analysis based on data-driven technology are inspired by contemporary achievement of deep learning for computer vision, natural language processing, and control [5,6,7]. Machine learning methods have been predominantly examined and investigated in multiple aspects of FEM. To predict steady flows in a representative, a capsule neural network was established in the area of fluid dynamics [8]. Besides, deep neural networks (DNN) also have been utilized to learn the structural features to boost the performance in topology optimization [9,10,11]. Furthermore, to estimate stress distribution, a fast deep learning approach is developed $[12,13]$. However, the generalization and therefore accuracy when presented with new data is compromised, since real world data could be vastly different from data used to train a surrogate model. On the other hand, to accelerate computing productivity, deep learning models $[14,15]$ are exploited to generate mesh automatically but without timesaving during the process of FEM calculation. Therefore, against the experimental success of traditional meshing meth- 

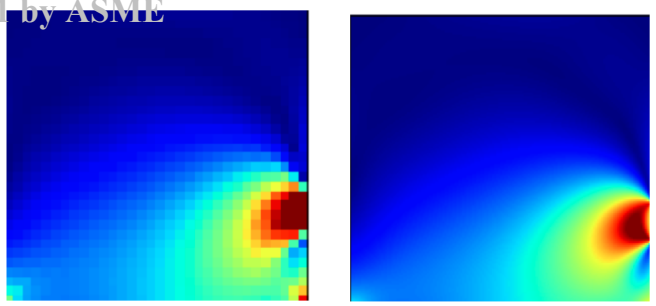

von Mises stress field

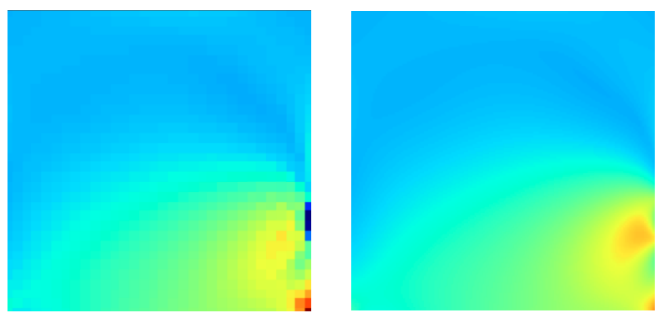

equivalent strain field

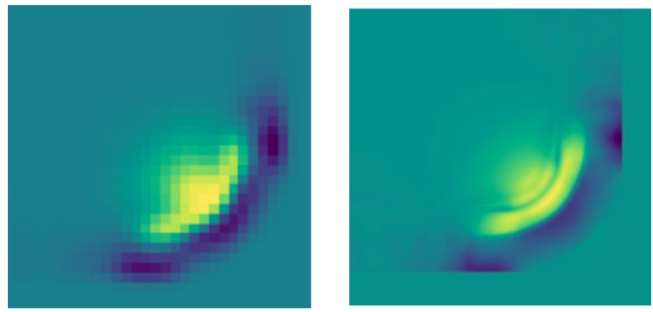

thinning field

Fig. 1. Physical fields computed by FEM in low $(32 * 32)$ and high (256*256) mesh density.

ods and machine learning methods, the following key challenges still persist. One of the drawbacks is lacking ability of generalization, which measures the performance of the model to learn finite samples of the data distribution to reconstruct other samples. Secondly, the computing time is inevitable to be tremendous while aiming to generate a high-quality outcome.

Inspired by the deep learning works on physical field prediction, a refined finite element simulation model based on a deep neural network is developed, named SuperMeshingNet. SuperMeshingNet is trained with low mesh-density FEM results as input which are corresponding to the high meshdensity FEM results as targets, while the same high meshdensity FEM results as outputs. SuperMeshingNet learns the mapping relationship from the low mesh-density to the high mesh-density, which can be utilized to instantaneously generate various version of higher mesh-density FEM results. To boost our approach, multiple deep learning techniques have been manipulated on SuperMeshingNet, in which ResUNet $[16,17]$ constitutes the main structure, the attention mechanism $[18,19,20]$ provides focus on training, and the perceptual feature $[21,22]$ promotes visual quality.

By using the proposed model, a variety of high mesh density finite element meshes can be calculated by only consuming computational resources on the order of minutes on generating lower mesh density finite element method results and model pre-training, which can immediately give feedback to the designer for saving time. Meanwhile, by assessing the test data set, which consists of distinctive data compared with the training set, the precision and the ability of generalization are validated. Moreover, the experiments validate SuperMeshingNet by competing for performance with baseline models at diverse scaling factors, including $2 \times, 4 \times$, and $8 \times$. SuperMeshingNet achieves superior performance on all used measure metrics, contrasted with the baseline approaches in the experiment. In addition, since deep learning network training is more dependent on matured learning infrastructures, the deep learning model migrates the calculation of finite element grids from traditional CPUs to cheaper GPUs, significantly reducing the computational cost.

Our main contributions are:

- A new method employing deep network models to generate high mesh-density simulation results through low meshdensity simulation results with seldom time-consuming.

- A new design of the output metrics measuring the quality of mapping relationship among various mesh-density scale of FEM results and evaluation matrices involving the geometric feature and perceptual feature of the FEM results.

- A hybrid neural network architecture with channel and spatial attention mechanism as the main structure of SuperMeshingNet that enable high accuracy.

\section{Related Work}

Mesh density expands the information of an FEM result and preserves physical field details which can only be captured, similar to an image super-resolution work that deep learning based approaches have become the dominant position since the advance of convolution neural network for processing image. Meanwhile, the attention mechanism that used in CNN achieves outstanding performance. This trend also shows in finite element analysis topics. Therefore, we review four parts of knowledge that related to our work: mesh density increasement, image-based super-resolution, attention mechanism in deep learning, and related knowledge about deep learning.

\subsection{Image Based Super-resolution}

Supervised neural networks have become state-of-theart work in super-resolution. In recent times, numerous works [21] have sought to improve the efficiency of learning pairs of LR-HR images by adopting sub-pixel convolutions. The first $\mathrm{CNN}$ architecture to recover high-resolution (HR) images by low-resolution (LR) images was proposed by Dong [23] named Super-resolution Convolutional Neural Network (SRCNN). Moreover, Lim. [24] applied ResNet to supersolution and brought substantial improvement compared with previous work that named Enhanced Deep Super-Resolution Residual Network (EDSR). By utilizing residual block, the depth of the network is increased with fewer extra parameters [16], which yields better performance. Furthermore, dense networks are used to boost detail, such as Residual Dense Network (RDN) [25]. Image resolution increasing 
Copyright and FEM meshing density improving face a key problem: completion of missing information. Therefore, drawing on the approach of image processing is of considerable significance to the information completion process in the finite element analysis process. The knowledge about image superresolution, such as texture feature, also benefits further FEM process.

From the aspect of human perception, texture usually describes the quality of the HR images. To reconstruct reasonable texture detail, an edge-directed Super-resolution (SR) algorithm integrated with the strengths of the detail synthesis approach was proposed by Tai et al. [26]. Capturing redundancies of similar image groups in various scopes by building a multi-scale dictionary shows the contribution of gaining texture detail [27]. Besides, Wang et al. employ spatial feature transform layers to generate more realistic and visually pleasing texture [28].

\subsection{Attention Mechanism}

Commonly, to inform the model where to focus attention can be claimed as an instruction to allocate inclinable available computing and storage resources towards the most descriptive components of source data with visual attention [18]. Newly, the attention mechanism has been applied to deep neural networks in some excellent works, covering natural language processing [29] and understanding in images [20]. To rebuild the feature and attention maps, these works are combined with a gating function [19].

The representation of interests is also improved by the attention mechanism. Providing appropriate attention to different regions, spatial locations and channels can acquire better performance. SelNet [30] attaches selected units at the end of CNN layers. These units control the feature information that can be passed to the next layer. Separately, the Residual Channel Attention Networks (RCAN) [31] focuses on channel attention. RCAN proposes RIR modules with a long skip connection to achieve channel attention which contains a similar structure with SqueezeNet. [32]. However, few techniques of attention mechanism have been developed to assist FEM.

\subsection{U-Net and ResNet}

Figure 2 shows the U-Net architecture [33], which connects the down-sampling layers and up-sampling layers at various resolutions to deliver context information in previous layers. To predict the missing information in lower resolution, the skip-connection structure reflects the input data to extrapolate missing context, which is important to the generator [34].

Deep network architectures have shown great contribution in performance, while they are difficult to train. A powerful design strategy is the concept of residual blocks [35]. The researchers claim that stacking layers by residual block should not damage the performance of network because the useless layers can be simplified with same performance [35].

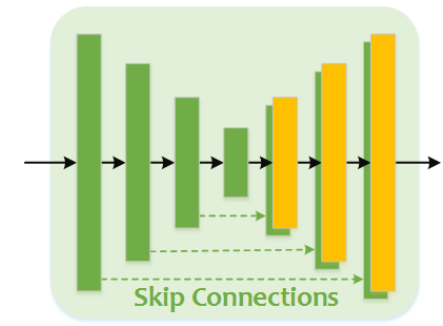

Fig. 2. Architecture of the U-Net. The skip connections between layers are illustrated as dotted arrows [17].

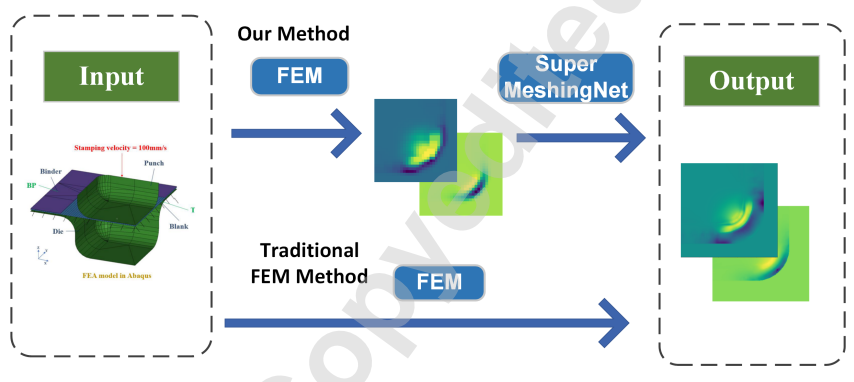

Fig. 3. Workflow of proposed method and traditional method.

\section{Technical Approach}

Fig. 3 presents the difference of our method and traditional method. Compared with traditional FEM method which only has one step to generate high mesh density physical field, our method contains two parts, using FEM to generate low mesh density physical field, and utilizing SuperMeshingNet on the FEM result, and therefore predict a high quality physical field. For both methods, boundary condition is input and high mesh density data is output. For SuperMeshingNet, the input source data set is represented as $x \in \mathbb{R}^{L \times W}$, and the target output data set is $y \in \mathbb{R}^{k L \times k W}$, where $L$ and $W$ donate the length and width of input data, and $k$ donates the scaling factor. The goal of the training process is to find the optimal weights $w$ in a neural network model $\boldsymbol{F}$, which performs as a nonlinear regression function $y=\boldsymbol{F}(x, w)$, where $x$ donates the low mesh-density data and $\boldsymbol{F}(x, w)$ represent the procedure of reconstructing high mesh-density data. To acquire the optimal weight $\boldsymbol{w}^{*}$, the distance between the target $y$ and the output of deep learning model $\boldsymbol{F}(x, w)$ is measured by Mean Absolute Error (MAE) and minimized as demonstrated in Eq. (1).

$$
\boldsymbol{w}^{*}=\arg \min _{w} \frac{1}{n} \sum_{i=1}^{n}\left|y_{i}-\boldsymbol{F}\left(x_{i}, w\right)\right|
$$

SuperMeshingNet is applied on the FEM to boost the mesh-density. For the purpose of reconstructing the high mesh-density data, the low mesh-density data is utilized for training the SuperMeshingNet model. 


\section{Copyright $3 . \mathbf{I}^{202}$ Network Architecture}

As shown in Fig. 4, the main structure of the model is composed of ResNet with the channel and spatial attention modules, and the down-sampling layer and the up-sampling layer are connected by a structure that named skip-connection.

At the beginning of the model, the input is up-sampled to the same size as the output through a bilinear interpolation method, which can maintain the symmetry of the convolution process and contribute to achieving skip-connections. Another benefit of applying the bilinear interpolation before convolution is avoiding a CNN-based up-sampled process, and optimizing the complexity of the model. Three scaling factors are also processed by interpolation, one linear interpolation operation can increase in sizes by two times. Therefore, scaling factors of $2 \times, 4 \times$, and $8 \times$ use $1,2,3$ bilinear interpolation layer respectively. The data at the beginning of down-sampling maintains the same size as the ground truth, which decreasing to a half through a Res + Attention module. Behind the four down-sampling Res + Attention modules, we added a Res34 structure, which supports the model to train extremely deep neural networks with more than 50 layers. The implementation of ResNet is able to avoid the notorious vanishing gradient problem during training and enhance the performance by increasing the network depth [35].

Up-sampling process appearances mirror the architecture of the down-sampling process. Besides, a geometric attention map that contains the information of geometric features generated from the training set is learned by a geometric feature extractor and added on the last third up-sampling module to highlight the geometric attention of the model on the image of the FEM results. Moreover, a perceptual feature extractor composed by ResNet is implemented to optimize the model by the perceptual loss $[36,37,38]$, which enhances the performance in feature space. To lighten the parameters of SuperMeshingNet and advance the training efficiency, two down-sampling layers are used by the geometric extractor and perceptual feature extractor.

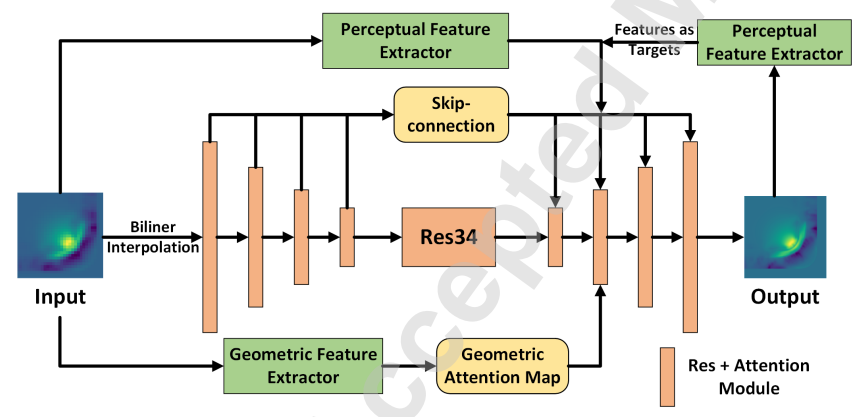

Fig. 4. Architecture of the SuperMeshingNet.

\subsection{Attention Module}

Channel and Spatial Attention Module. To increase the representation power, the model focuses on important features and suppresses unnecessary ones by varying the weights of channels and spatial features. The attention approach is integrated into the two ResBlocks. The overall explanation of the attention module constructed with ResBlock is shown in Fig. 5. The channel and spatial attention can change the weight of different channels and spatial during the training. Therefore, the channel and spatial that contribute to accuracy are located higher weights, improving training efficiency and boosting performance. The feature map can be defined as $F \in \mathbb{R}^{C \times H \times W}$. Subsequently, the 1D channel attention map and the 2D spatial channel attention map are characterized as $M_{c} \in \mathbb{R}^{C \times 1 \times 1}$ and $M_{s} \in \mathbb{R}^{1 \times H \times W}$, respectively.

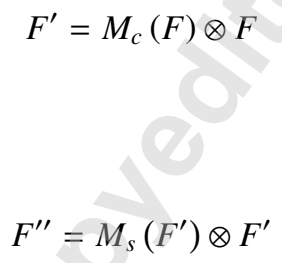

where $\otimes$ is element-wise multiplication. As shown in Fig. 5 , the final clarified output $F^{\prime \prime}$ comes from the model with channel attention value and spatial attention value multiplied.

The channel attention module detects the meaningful features of a given input [39]. The previous implementation also proves that the representation ability of networks can be promoted significantly by appealing both average-pooled and max-pooled features simultaneously [40]. Average pooling and max pooling are sample-based discretization process, in which average pooling [41] uses average value of the filter while max pooling [42] uses max value. Therefore, we employ both technologies in channel attention:

$$
M_{c}(F)=\sigma\left(W_{1}\left(W_{0}\left(F_{\text {avg }}^{c}\right)\right)+W_{1}\left(W_{0}\left(F_{\text {max }}^{c}\right)\right)\right)
$$

where the sigmoid function is represented as $\sigma, F_{\max }^{c}$ and $F_{\text {avg }}^{c}$ are the max-pooled features and average-pooled features. $W_{0}$ and $W_{1}$ share the weights for inputs followed by Leaky ReLU activation function $\sigma$.

Dissimilar with the channel attention mechanism, the spatial attention mechanism pays more attention to the location of the effective informative part. After two pooling operations on the feature map, there are two 2D maps: $F_{\text {avg }}^{s} \in \mathbb{R}^{1 \times H \times W}$ and $F_{\text {max }}^{s} \in \mathbb{R}^{1 \times H \times W}$. Subsequently, a standard convolution layer $f$ with a $7 \times 7$ kernel is utilized to process the concatenated inputs and followed by $\sigma$. The final refined output can be described as:

$$
M_{s}(F)=\sigma\left(f^{7 \times 7}\left(\left[F_{\text {avg }}^{s} ; F_{\text {max }}^{s}\right]\right)\right)
$$

Physical Attention Map At the same time, we find that when FEM is applied in the physical simulation process, the geometric shape has a salient feature that is easily observed 


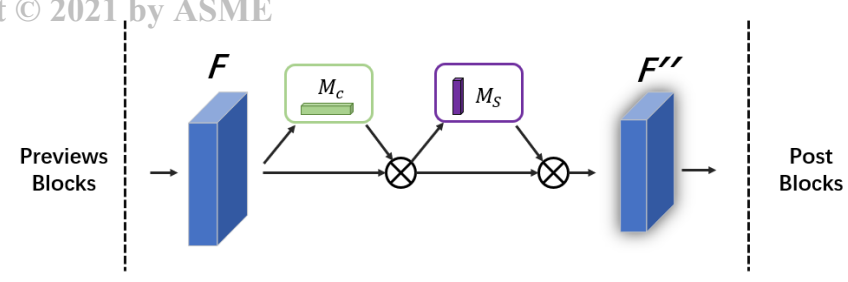

Fig. 5. Implement of Res + Attention Module.

as shown in Fig. 6. These positions are often places where the value changes drastically, increasing the difficulty of prediction and decreasing in global accuracy. Therefore, features related to geometry should be highlighted to assist the training and enhance the performance.

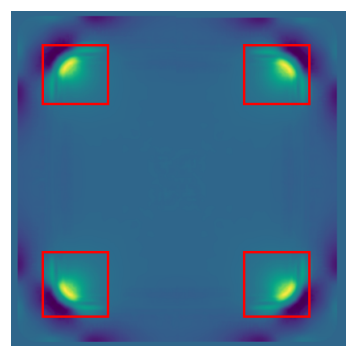

Fig. 6. The geometric feature shown in FEM result of stamping.

Meanwhile, from the visualization aspect, the texture and edge in physical field can be the area that fluctuate in value dramatically. These special areas contain more complicate physical features compared with flat area.

To highlight the physical feature, we employ the perceptual loss, which enhance the performance and compute in latent feature level, to improve perceptual quality. On the other hand, the geometric feature map represents the performance of geometric attention on the training set, and in the reconstruction experiment after training, then the attention map will no longer need to be optimized.

\subsection{Loss Function}

The loss function of our approach contains three parts, which can be interpreted as:

$$
\mathcal{L}_{\text {overall }}=\lambda_{c} \mathcal{L}_{c}+\lambda_{p} \mathcal{L}_{p}+\lambda_{g} \mathcal{L}_{g}
$$

where $\mathcal{L}_{c}, \mathcal{L}_{p}$ and $\mathcal{L}_{g}$ denote the content loss, perceptual loss and geometric loss respectively. $\lambda_{c}, \lambda_{p}$ and $\lambda_{g}$ denote the loss coefficient of each part of the loss function.

Content Loss. The first and the most important part of the overall loss is content loss which describes the quality of reconstruction. The $\mathcal{L}_{c}$ Loss [43] is described as:

$$
\mathcal{L}_{c}=\frac{1}{L \times W \times N} \sum_{i=1}^{N}\left|x_{i}-y_{i}\right|
$$

where $L$ and $W$ donate the size of the data, $N$ means the batch size. $x, y$ represents the high mesh-density data generated by our model and ground truth, respectively. The $\mathcal{L}_{1}$ Loss has been indicated to be sharper for performance and more accessible for convergence compared to $\mathcal{L}_{2}$ Loss. Therefore, we choose $\mathcal{L}_{1}$ Loss to optimize the content performance.

Perceptual Loss. Some works $[36,37,38]$ in image super-resolution have adopted perceptual loss, which has been proved effective in improving quality in edge and texture, where is hard to reconstruct. To advance the local performance, we address the perceptual feature and enhance the similarity in feature space. We take advantage of perceptual loss, supplementing the loss function in feature space. The perceptual loss can be described as:

$$
\mathcal{L}_{p}=\frac{1}{L_{i} \times W_{i} \times N}\left|\phi_{i}^{N N}(x)-\phi_{i}^{N N}(y)\right|^{2}
$$

where the $L_{i}, W_{i}$ are the shape of the feature map $\phi_{i}^{N N}$ in the $i$-th layer's of neural network $(N N)$ model. As shown in Fig. 4 , the target feature map $\phi_{i}^{N N}(y)$ are acquired by perceptual feature extractor that is composed by three Res + Attention modules, and the input feature map $\phi_{i}^{N N}(x)$ comes from the up-sampling process. Here the perceptual loss constraints the predicted high-resolution data to have similar detail to the ground truth in feature dimension, such as flatness and texture of the output.

Geometric Loss. FEM is a simulation of the objective physical world, so its geometric features are salient features that can be observed by humans. We extract obvious geometric features by adding geometric attention to the model and apply them. In order to optimize the geometric attention map, we introduced geometric loss combining the Kullback-Leibler divergence [44] loss:

$$
\mathcal{L}_{g}=\frac{1}{L \times W \times N} \sum_{i=1}^{N} y_{i}\left(\log y_{i}-\log x_{i}\right)
$$

where $y_{i}$ comes from the distribution of the ground truth, $x_{i}$ is acquired by the distribution of geometric extractor.

\section{Experiments}

The proposed SuperMeshingNet and the control group are trained on the same data set that contains aligned low mesh-density data and high mesh-density data. Three interpolation methods (linear, quadratic, cubic) are implemented as 

perMeshingNet. We publicly share our work with full detail of implementation at https://github.com/zhenguonie/ 2021_SuperMeshing_2D_Metal_Forming.

\subsection{Data set}

In this study, the data set is comprised of the thinning fields of the cold stamping process, generated by PAMSTAMP respectively with low and high mesh-density. As the symmetry of the simulation component, shown in Fig. 6, Figure 7 displays the data for the experiment, which is constructed by a quarter of the simulation result, and as-formed components expand into 2D images. There are 1,980 cases in the training set and 220 cases in the test set, which differ in the size of $C 1, C 2, C 3$, the curvature of $R 1, R 2, R 3$, and stamping velocity as shown in Fig. 7. The top view of data is shown in Fig. 8. The stamping direction is along the $z$ axis with stamping velocity set to $100 \mathrm{~mm} / \mathrm{s}$. All the thinning value are normalized in $(0,1)$.

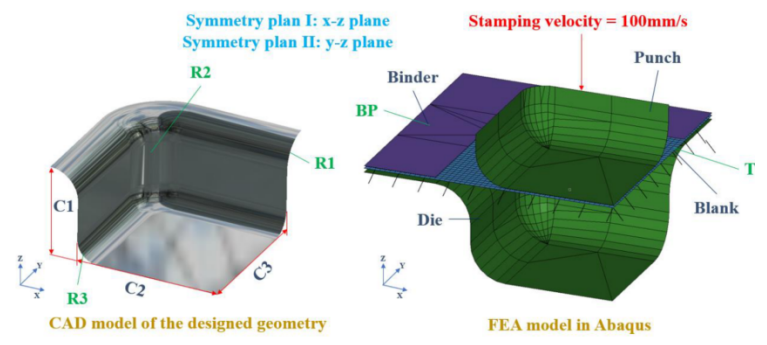

Fig. 7. The CAD model and FEM model of a sample metal forming simulation [45].

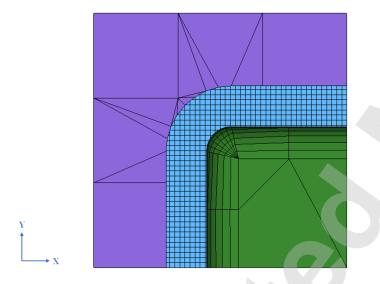

Fig. 8. The top view FEM model of a sample metal forming simulation [45].

\subsection{Evaluation Metrics}

To evaluate the model, we use MAE shown in Eq.(10) and MSE shown in Eq.(11) for measuring the divergence between the predicted high mesh-density data and the ground truth.

$$
\mathrm{MAE}=\frac{1}{n} \sum_{i=1}^{n}\left|y^{(i)}-\hat{y}^{(i)}\right|
$$

where $n$ represents the number of test data, $y$ donates the real value of the physical field, and $\hat{y}$ donates the predicted value of the physical field. Meanwhile, the distribution of the MAE and MSE of test cases are presented, which explains the performance at the level of the single case.

Moreover, some state-of-art processing results are exhibited to demonstrate the superior performance of the reconstruction process.

\section{Results and Discussions}

Our model is implemented by PyTorch, and trained on GPU (NVIDIA GeForce GTX 2080Ti). Adam optimizer [46] is used to train the model for optimization. To convince the achievement of SuperMeshingNet, three aspects of the result is shown and discussed. Firstly, we present the performance of SuperMeshingNet that is trained with the data set on various test cases to examine the effectiveness and generalization of our model. Subsequently, the performance of SuperMeshingNet, various deep learning models with different architecture, and three interpolation methods are compared and discussed. Finally, aiming to explain the ability of computation resource-saving, the computing time under the same scenario is collected and presented.

\subsection{Model Evaluation}

As shown in Fig. 9, the loss trend of SuperMeshingNet in training, the validation loss restrains at 800 epochs, and train loss convergence after 1000 epochs, which proves that SuperMeshingNet performs reasonable convergence ability and indicates that the state-of-art training strategy should stop at 600 epochs to avoid over-fitting.

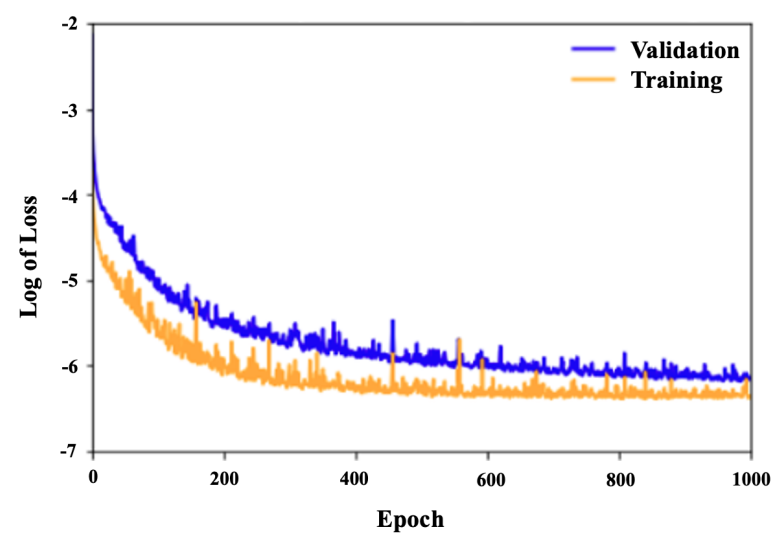

Fig. 9. The loss of the SuperMeshingNet during training. 


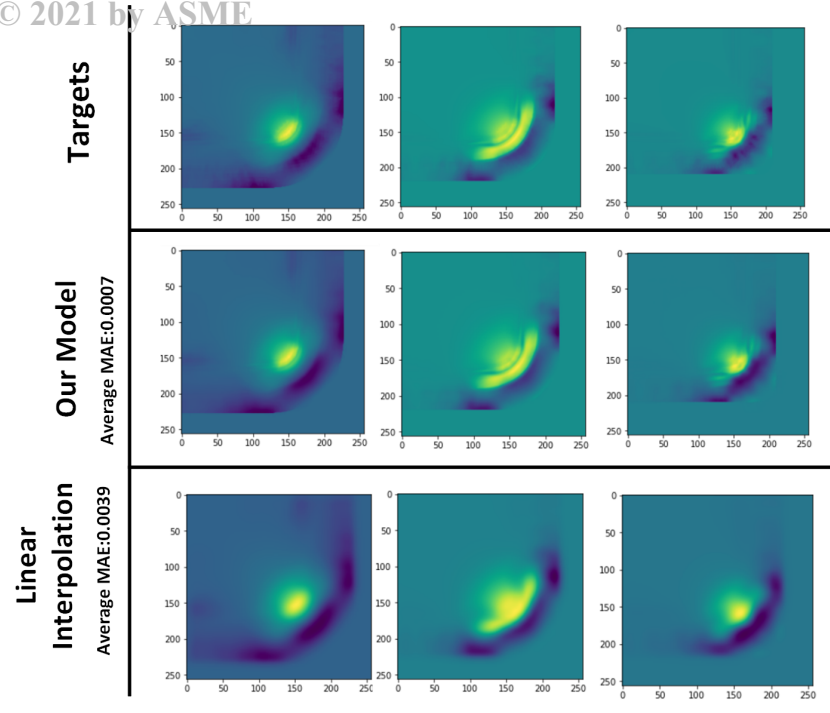

Fig. 10. Samples comparing result of our model with target and linear interpolation.

After completing the training of the model, we observe its performance in the form of pictures on shape and texture and compare the results of the model reconstruction with the results obtained by the linear interpolation approach and the results obtained by the finite element calculation (ground truth). Figure 10 displays three cases from each method, where our model is SuperMeshingNet. Both our model and linear interpolation use the FEM result with the size of $32 \times 32$ as input to reconstruct an $8 \times$ larger contemporary image which shape is $256 \times 256$. From the picture, it is apparent that although the linear interpolation method can retain part of the shape information in the target data, the overall performance has been completely roughened due to the inaccuracy of details and textures, and the result is quite blurred, which is obviously not able to provide the further application. On the contrary, our model's results of reconstruction are consistent with the ground truth in terms of texture and shape, as well as the values. Compared with the linear interpolation average MAE error, the analytical product of our model is six times as low as, reaching 0.0007 . The analytical results are similar to FEM calculations, which can predict the distribution trend of the value, and the error is minimal, so from the user's point of view, it will not affect the prediction of the thinning field trend.

A more visual comparison is presented in Fig. 11, where various examples are reconstructed by our model to manifest the high quality of the outcomes. These examples include the $32 \times 32$ sizes to $2 \times, 4 \times$, and $8 \times$ mesh density of growth process The results suggest that our model can match the true value with their respective reconstruction methods in the process of boosting the mesh density of the input data. Therefore, the final $8 \times$ reconstruction results based on all methods perfectly fit the fundamental values in terms of overall details.

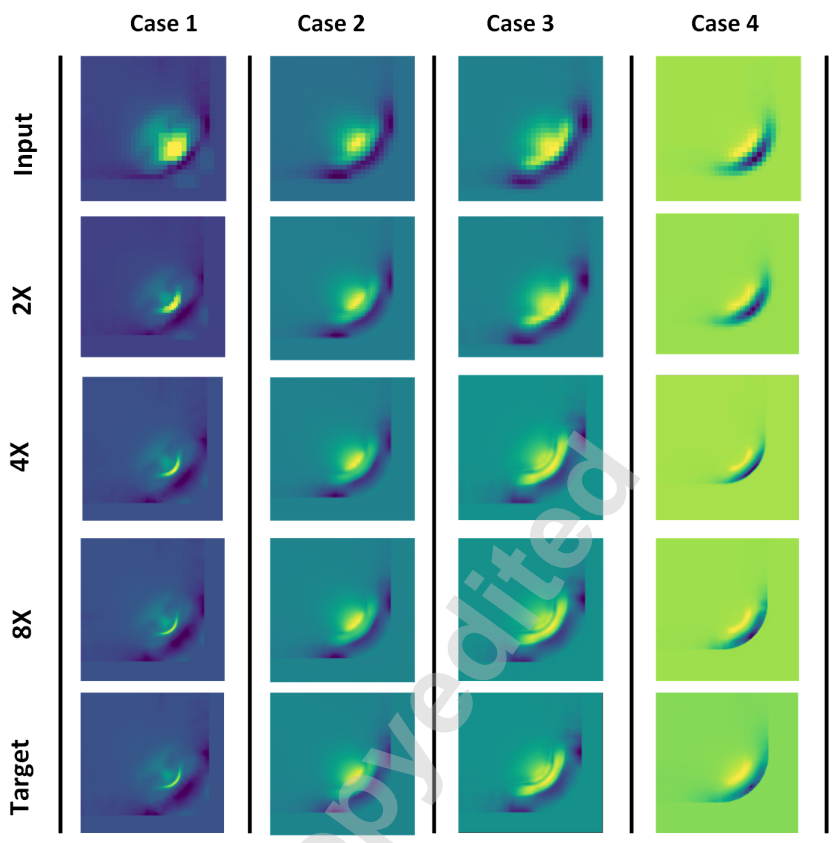

Fig. 11. Cases randomly selected from the test data set.

\subsection{Accuracy and Performance}

In this section, we verify the effectiveness of SuperMeshingNet on various scaling factors through comparing seven techniques, including baseline 1 - linear interpolation, baseline 2 - quadratic interpolation, baseline 3 - cubic interpolation, ResNet, ResNet with skip-connection (ResUNet), ResUNet with attention mechanism, and SuperMeshingNet. In addition, the accuracy is presented by comparing various models. SuperMeshingNet contains five parts: skip-connection, attention module, perception feature extractor for feature synthesis, channel and spatial attention module, and the geometric feature attention map. The ablation of the experiment also can be suggested in Tab. 1. After the skip-connection is employed, the MAE dropped to $4.758 \times 10^{-4}$ from $6.495 \times 10^{-4}$ when the scaling factor is 2 . When channel and spatial attention module are utilized, the MAE can be decreased to $4.152 \times 10^{-4}$, which verifies the effectiveness of the attention mechanism. Subsequently, when the feature synthesis and geometric feature attention are progressively added, perceptual features will be enhanced, amplifying the performance to $3.203 \times 10^{-4}$. Furthermore, the similar trend can be summarized from the scaling factor of $4 \times$ and $8 \times$.

Figure 12 illustrates the distribution of MSE after figuring out all test cases. Contrasting the cases acquired by linear interpolation in Fig. $12(\boldsymbol{d})$, in which most cases range in $10^{-4}$ to $3 \times 10^{-3}$, the deep learning models constrain MSE lower than $1 \times 10^{-5}$, proving that the deep learning methods perform more outstanding outcome compared with traditional linear interpolation. Furthermore, from Fig. 12 (a), except for a few exceptions, SuperMeshingNet restricts the MES of three-quarters test set to be lower than $10^{-6}$. At the same time, other cases tend to be lower than ResUNet with attention mechanism in Fig. $12(\boldsymbol{b})$ and ResNet in Fig. $12(\boldsymbol{c})$, convincing the superior generalization of SuperMeshingNet. 


\begin{tabular}{c|ccc}
\hline Model & $8 \times$ & $4 \times$ & $2 \times$ \\
\hline Baseline 1 & 76.94 & 35.81 & 12.71 \\
Baseline 2 & 76.46 & 34.98 & 12.32 \\
Baseline 3 & 76.91 & 35.75 & 12.83 \\
ResNet & 9.714 & 7.923 & 6.495 \\
Res+U & 7.770 & 6.174 & 4.758 \\
Res+ $U+A$ & 5.957 & 5.412 & 4.152 \\
SMNet & 5.465 & 4.682 & 3.203 \\
\hline
\end{tabular}

parison results demonstrate the superiority of our proposed SuperMehsingNet over the other approaches.

\subsection{Computation Allocation}

Another superior character of our model is time-saving. For each FEM calculation which the mesh density is $256 \times 256$ in PAM-STAMP, $T_{F 256}$ spends 15.21 seconds on CPU (R7$4800 \mathrm{HS}$ ), but only 0.41 seconds is cost by FEM computation with the size of $32 \times 32, T_{F 32}$. For SuperMeshingNet. With the trained model, the time required to reconstruct the low meshdensity data to high mesh-density is extremely small, $T_{r}$, only 0.012 seconds is spent. Our method $T_{S}$ and traditional FEM method $T_{F}$ shown in Fig. 3 can be claimed as:

$$
T_{S}=N \times T_{r}+N \times T_{F 32}
$$

metrics used is MAE. Baseline 1 represents the linear interpolation, Baseline 2 represents the quadratic interpolation, Baseline 3 represents the cubic interpolation, $U$ is the skip-connection structure, $A$ is the attention module.

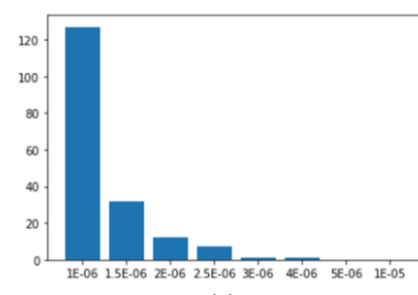

(a)

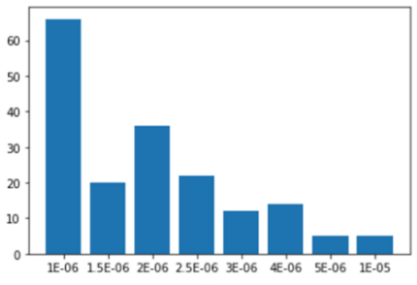

(c)

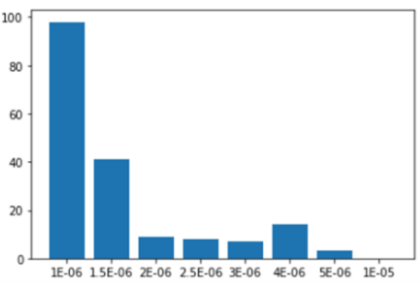

(b)

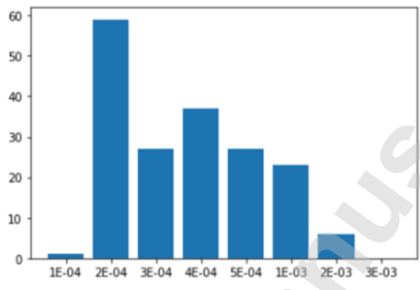

(d)

Fig. 12. The distribution of the MSE of the four models, $\boldsymbol{a}$ : SuperMeshingNet, $\boldsymbol{b}$ : ResUNet+Attention Mechanism, $\boldsymbol{c}:$ ResNet, and $\boldsymbol{d}$ : linear interpolation.

The complementary justification is demonstrated in Tab. 2, which shows both MAE and MSE of five techniques on training and test set, including interpolation methods, SRCNN [23], EDSR [24], RDN [25] and SuperMeshingNet. SRCNN, EDSR and RDN are three state-of-art super-resolution networks. All experiments in Tab. 2 are performed with a scaling factor of $2 \times$ low mesh-density and high mesh-density data pair. SuperMeshingNet performs the lowest error. As displayed in the comparison results, SuperMeshingNet significantly outperforms both MAE and MSE on training and test data sets than popular super-resolution techniques. Other super-resolution works focus on image quality from human perceptual that measured by SSIM and PSNR rather than the value prediction. Therefore, these methods perform poorly in physical filed value prediction. The MAE and MSE com-

where $N$ donates the number of data to be processed, which is named workload. In addition, because SuperMeshingNet breaks the limitations of standard finite element calculations on the CPU and migrates it to the GPU-based deep learning framework for calculation. Therefore, the cost required for calculation is reduced because of time and the reduction in equipment prices.

Tab. 3 shows the performance of the time. As shown in Tab. 3, with the continuous increase of processing tasks, it can clearly be seen that the time of traditional finite element calculation are at a considerable disadvantage. Therefore, using the low mesh-density finite element analysis result under the condition of ensuring absolute accuracy and then reconstructing it to high mesh-density using SuperMeshingNet can significantly reduce the time and cost of the entire finite element analysis process.

\subsection{Limitations and Future Work}

SuperMeshingNet has an excellent performance in automatically improving the density of finite element analysis. This technology can significantly reduce the calculation time and cost while maintaining a low MAE and extreme deviation. However, future works can still be extended.

The Transformer [47] structure with attention as the main structure has developed rapidly, and critical applications have been born in various fields, including the super-resolution field $[48,49]$. The complete Transformer structure can be utilized to build the model and promote model innovation. In addition, though SuperMeshingNet accomplishes superior performance on 2D data with a regular grid, solving the problem of unstructured mesh which consumes more computing resources is valuable as a future direction. To reconstruct unstructured mesh and 3D data, it is appropriate to replace the network architecture with a graph neural network (GNN), which will be more applicable on the 3D data. 
Table 2. Result comparison of the super-resolution methods with SuperMeshingNet

\begin{tabular}{|c|c|c|c|c|c|}
\hline \multicolumn{2}{|c|}{ Metrics } & \multicolumn{2}{|c|}{ MAE } & \multicolumn{2}{|c|}{ MSE } \\
\hline No. & Model & Training & Test & Training & Test \\
\hline 1 & Baseline 1 & $1.256 \times 10^{-3}$ & $1.271 \times 10^{-3}$ & $1.753 \times 10^{-5}$ & $1.783 \times 10^{-5}$ \\
\hline 2 & Baseline 2 & $1.224 \times 10^{-3}$ & $1.232 \times 10^{-3}$ & $2.078 \times 10^{-5}$ & $2.068 \times 10^{-5}$ \\
\hline 3 & Baseline 3 & $1.268 \times 10^{-3}$ & $1.283 \times 10^{-3}$ & $1.817 \times 10^{-5}$ & $1.808 \times 10^{-5}$ \\
\hline 4 & SRCNN & $5.341 \times 10^{-4}$ & $5.792 \times 10^{-4}$ & $1.072 \times 10^{-6}$ & $1.476 \times 10^{-6}$ \\
\hline 5 & EDSR & $5.428 \times 10^{-4}$ & $5.432 \times 10^{-4}$ & $4.403 \times 10^{-6}$ & $4.406 \times 10^{-6}$ \\
\hline 6 & RDN & $5.496 \times 10^{-4}$ & $5.650 \times 10^{-4}$ & $3.611 \times 10^{-6}$ & $4.187 \times 10^{-6}$ \\
\hline 7 & SMNet & $3.145 \times 10^{-4}$ & $3.203 \times 10^{-4}$ & $4.495 \times 10^{-7}$ & $5.138 \times 10^{-7}$ \\
\hline
\end{tabular}

Note: Baseline 1 represents the linear interpolation, Baseline 2 represents the quadratic interpolation, Baseline 3 represents the cubic interpolation; SRCNN [23], EDSR [24], and RDN [25] are prior and superior super-resolution networks; SMNet is SuperMeshingNet.

Table 3. The time and cost on different workload

\begin{tabular}{ccc}
\hline & SuperMeshingNet & FEM \\
\hline$N \times$ Size & Time $(\mathrm{s})$ & Time(s) \\
\hline $1 \times(256,256)$ & 0.422 & 15.21 \\
$10^{2} \times(256,256)$ & 42.2 & $1.52 \times 10^{3}$ \\
$10^{4} \times(256,256)$ & $4.22 \times 10^{3}$ & $1.52 \times 10^{5}$ \\
\hline
\end{tabular}

\section{Conclusions}

We present a new deep learning model named SuperMeshingNet to reconstruct the FEM outcomes from low meshdensity to high mesh-density in physical fields. With the trained model, the high mesh-density results can be produced immediately with high accuracy, contributing to the efficiency of FEM in physical fields.

SuperMeshingNet draws the self-attention module and perceptual features, and employs the deepened ResNet and skip-connections to boost the model performance. Integrated with these gainful techniques, SuperMeshingNet is proven to be constructive to outperform the three baseline models and other Super-resolution models in generalization and accuracy by comparative experiments. Extensive experiments demonstrate the superiority of our work when compromising scaling factors of $2 \times, 4 \times$, and $8 \times$. Concurrently, compared with the FEM computation, our model can effectively save computational time and cost. Embedding the proposed model into FEM, our approach can ensure expeditious computational process and marvelous spatial precision simultaneously under multiple scaling factors for high mesh-density.

\section{Acknowledgement}

This work is supported by National Key R\&D Program of China under Grant 2019YFA0706701.

\section{References}

[1] Reddy, J. N., 1996. "Finite element procedures. kj bathe.". APPLIED MECHANICS REVIEWS(11), p. B117.

[2] Li, N., Lin, J., Dean, T. A., Dry, D., and Balint, D., 2014. "Materials modelling for selective heating and press hardening of boron steel panels with graded microstructures.". Procedia Engineering, 81(11th International Conference on Technology of Plasticity, ICTP 2014, 19-24 October 2014, Nagoya Congress Center, Nagoya, Japan), pp. 1675 - 1681.

[3] Nicholson, D. W., 2008. Finite element analysis : thermomechanics of solids. CRC Press.

[4] Cai, H., 2020. "A fast calculation method for steady state performance of high speed traction induction machine by finite element analysis.”. 2020 IEEE Energy Conversion Congress and Exposition (ECCE), Energy Conversion Congress and Exposition (ECCE), 2020 IEEE, pp. $4284-4291$.

[5] Krizhevsky, A., Sutskever, I., and Hinton, G. E., 2017. "Imagenet classification with deep convolutional neural networks.". Communications of the ACM, 60(6), pp. 84 -90 .

[6] Devlin, J., Chang, M. W., Lee, K., and Toutanova, K., 2018. "Bert: Pre-training of deep bidirectional transformers for language understanding." Arxiv.

[7] Silver, D., Schrittwieser, J., Simonyan, K., Antonoglou, I., Huang, A., Guez, A., Hubert, T., Baker, L., Lai, M., Bolton, A., Chen, Y., Lillicrap, T., Hui, F., Sifre, L., van den Driessche, G., Graepel, T., and Hassabis, 
Copyright (C) 2020 D., 201\%. Mastering the game of go without human knowledge.". Nature, 550(7676), p. 354.

[8] Kurtakoti, A. U., and Chickerur, S., 2020. "Steady flow approximation using capsule neural networks.". 2020 IEEE Sixth International Conference on Multimedia Big Data (BigMM), Multimedia Big Data (BigMM), 2020 IEEE Sixth International Conference on, pp. $257-261$.

[9] Oh, S., Jung, Y., Kim, S., Lee, I., and Kang, N., 2019. "Deep generative design: Integration of topology optimization and generative models.". Arxiv.

[10] Rawat, S., and Shen, M. H. H., 2019. "A novel topology optimization approach using conditional deep learning.". Arxiv.

[11] Nie, Z., Lin, T., Jiang, H., and Kara, L. B., 2020. “Topologygan: Topology optimization using generative adversarial networks based on physical fields over the initial domain.". Arxiv.

[12] Liang, L., Liu, M., Martin, C., and Sun, W., 2018. “A deep learning approach to estimate stress distribution: a fast and accurate surrogate of finite-element analysis.”. JOURNAL OF THE ROYAL SOCIETY INTERFACE, 15(138).

[13] Jiang, H., Nie, Z., Yeo, R., Farimani, A. B., and Kara, L. B., 2020. "Stressgan: A generative deep learning model for $2 \mathrm{~d}$ stress distribution prediction.". The American Society of Mechanical Engineers, Journal od Applied Mechanics.

[14] Feng, Y., Feng, Y., You, H., Zhao, X., and Gao, Y., 2018. "Meshnet: Mesh neural network for 3d shape representation.". Arxiv.

[15] Pan, J., Li, J., Han, X., and Jia, K., 2018. "Residual meshnet: Learning to deform meshes for single-view $3 \mathrm{~d}$ reconstruction.". 2018 International Conference on $3 D$ Vision (3DV), 3D Vision (3DV), 2018 International Conference on, $3 D V$, pp. $719-727$.

[16] He, K., Zhang, X., Ren, S., and Sun, J., 2015. "Deep residual learning for image recognition". 2016 IEEE Conference on Computer Vision and Pattern Recognition (CVPR), Computer Vision and Pattern Recognition (CVPR), 2016 IEEE Conference on.

[17] Nie, Z., Jiang, H., and Kara, L. B., 2020. "Stress field prediction in cantilevered structures using convolutional neural networks.". JOURNAL OF COMPUTING AND INFORMATION SCIENCE IN ENGINEERING, 20(1).

[18] Cao, C., Liu, X., Yang, Y., Yu, Y., Wang, J., Wang, Z., Huang, Y., Wang, L., Huang, C., Xu, W., Ramanan, D., and Huang, T. S., 2015. Look and think twice: Capturing top-down visual attention with feedback convolutional neural networks.

[19] Li, K., Wu, Z., Peng, K.-C., Ernst, J., and Fu, Y., 2018. "Tell me where to look: Guided attention inference network.”. 2018 IEEE/CVF Conference on Computer Vision and Pattern Recognition, Computer Vision and Pattern Recognition (CVPR), 2018 IEEE/CVF Conference on, CVPR, pp. 9215 - 9223.

[20] Elakkiya, E., Selvakumar, S., and Leela Velusamy, R., 2020. "Textspamdetector: textual content based deep learning framework for social spam detection using con- joint attention mechanism.”. Journal of Ambient Intelligence and Humanized Computing, p. 1.

[21] Shi, W., Caballero, J., Huszar, F., Totz, J., Aitken, A. P., Bishop, R., Rueckert, D., and Wang, Z., 2016. "Real-time single image and video super-resolution using an efficient sub-pixel convolutional neural network.". 2016 IEEE Conference on Computer Vision and Pattern Recognition (CVPR), Computer Vision and Pattern Recognition (CVPR), 2016 IEEE Conference on, pp. $1874-1883$.

[22] Ledig, C., Theis, L., Huszar, F., Caballero, J., Cunningham, A., Acosta, A., Aitken, A., Tejani, A., Totz, J., Wang, Z., and Shi, W., 2017. "Photo-realistic single image super-resolution using a generative adversarial network". Arxiv.

[23] Yoon, Y., Jeon, H.-G., Yoo, D., Lee, J.-Y., and Kweon, I. S., 2015. "Learning a deep convolutional network for light-field image super-resolution.”. 2015 IEEE International Conference on Computer Vision Workshop (ICCVW), Computer Vision Workshop (ICCVW), 2015 IEEE International Conference on, Computer Vision Workshops (ICCVW), 2013 IEEE International Conference on, pp. $57-65$.

[24] Lim, B., Son, S., Kim, H., Nah, S., and Lee, K. M., 2017. "Enhanced deep residual networks for single image super-resolution.”. 2017 IEEE Conference on Computer Vision and Pattern Recognition Workshops (CVPRW), Computer Vision and Pattern Recognition Workshops (CVPRW), 2017 IEEE Conference on, CVPRW, pp. 1132 $-1140$.

[25] Xu, J., Chae, Y., Stenger, B., and Datta, A., 2018. "Dense bynet: Residual dense network for image super resolution.". 2018 25th IEEE International Conference on Image Processing (ICIP), Image Processing (ICIP), 2018 25th IEEE International Conference on, pp. 71 75.

[26] Yu-Wing, T., Shuaicheng, L., Brown, M., and Lin, S., 2010. "Super resolution using edge prior and single image detail synthesis.". 2010 IEEE Computer Society Conference on Computer Vision and Pattern Recognition, Computer Vision and Pattern Recognition (CVPR), 2010 IEEE Conference on, pp. 2400 - 2407.

[27] Kaibing, Z., Xinbo, G., Dacheng, T., and Xuelong, L., 2012. "Multi-scale dictionary for single image superresolution.". 2012 IEEE Conference on Computer Vision and Pattern Recognition, Computer Vision and Pattern Recognition (CVPR), 2012 IEEE Conference on, pp. $1114-1121$.

[28] Wang, X., Yu, K., Dong, C., and Change Loy, C., 2018. "Recovering realistic texture in image super-resolution by deep spatial feature transform.". 2018 IEEE/CVF Conference on Computer Vision and Pattern Recognition, Computer Vision and Pattern Recognition (CVPR), 2018 IEEE/CVF Conference on, CVPR, pp. 606-615.

[29] An, F.-P., and Liu, J.-e., 2021. "Medical image segmentation algorithm based on multilayer boundary perception-self attention deep learning model.". Multimedia Tools and Applications: An International Journal, 
[30] Choi, J.-S., and Kim, M., 2017. "A deep convolutional neural network with selection units for superresolution.". 2017 IEEE Conference on Computer Vision and Pattern Recognition Workshops (CVPRW), Computer Vision and Pattern Recognition Workshops (CVPRW), 2017 IEEE Conference on, CVPRW, pp. 1150 $-1156$.

[31] Zhang, Y., Li, K., Li, K., Wang, L., Zhong, B., and $\mathrm{Fu}, \mathrm{Y}$., 2018. "Image super-resolution using very deep residual channel attention networks.". Arxiv.

[32] Iandola, F. N., Han, S., Moskewicz, M. W., Ashraf, K., Dally, W. J., and Keutzer, K., 2016. "Squeezenet: Alexnet-level accuracy with 50x fewer parameters and $<0.5 \mathrm{mb}$ model size.". Arxiv.

[33] Long, J., Shelhamer, E., and Darrell, T., 2015. "Fully convolutional networks for semantic segmentation". In Proceedings of the IEEE conference on computer vision and pattern recognition, pp. 3431-3440.

[34] Ronneberger, O., Fischer, P., and Brox, T., 2015. "U-net: Convolutional networks for biomedical image segmentation". In International Conference on Medical image computing and computer-assisted intervention, Springer, pp. 234-241.

[35] He, K., Zhang, X., Ren, S., and Sun, J., 2016. "Deep residual learning for image recognition.”. 2016 IEEE Conference on Computer Vision and Pattern Recognition (CVPR), Computer Vision and Pattern Recognition (CVPR), 2016 IEEE Conference on, pp. 770 - 778.

[36] Johnson, J., Alahi, A., and Fei-Fei, L., 2016. "Perceptual losses for real-time style transfer and super-resolution.". In Lecture notes in computer science, p. 694.

[37] Zhang, Z., Wang, Z., Lin, Z., and Qi, H., 2019. "Image super-resolution by neural texture transfer.". 2019 IEEE/CVF Conference on Computer Vision and Pattern Recognition (CVPR), Computer Vision and Pattern Recognition (CVPR), 2019 IEEE/CVF Conference on, pp. $7974-7983$.

[38] Ledig, C., Theis, L., Huszar, F., Caballero, J., Cunningham, A., Acosta, A., Aitken, A., Tejani, A., Totz, J., Wang, Z., and Shi, W., 2016. "Photo-realistic single image super-resolution using a generative adversarial network.". Arxiv.

[39] Park, J., Woo, S., Lee, J.-Y., and Kweon, I. S., 2018. "Bam: Bottleneck attention module.". Arxiv.

[40] Woo, S., Park, J., Lee, J.-Y., and Kweon, I. S., 2018. "Cbam: Convolutional block attention module.". In Lecture notes in computer science, p. 3.

[41] Chu, T., Chen, Y., Huang, L., Tan, H., Cao, J., and Xu, Z., 2020. "Street view image retrieval with average pooling features.". IGARSS 2020 - 2020 IEEE International Geoscience and Remote Sensing Symposium, Geoscience and Remote Sensing Symposium, IGARSS 2020 - 2020 IEEE International, pp. 1205 - 1208.

[42] Bachtiar, Y., and Adiono, T., 2019. "Convolutional neural network and maxpooling architecture on zynq soc fpga.". 2019 International Symposium on Electronics and Smart Devices (ISESD), Electronics and Smart De- vices (ISESD), 2019 International Symposium on, pp. 1 -5 .

[43] Janocha, K., and Czarnecki, W. M., 2017. "On loss functions for deep neural networks in classification.". Arxiv.

[44] Zhao, P., and Lai, L., 2020. "Minimax optimal estimation of kl divergence for continuous distributions.". IEEE Transactions on Information Theory, Information Theory, IEEE Transactions on, IEEE Trans. Inform. Theory, 66(12), pp. $7787-7811$.

[45] Zhou, H., Xu, Q., and Li, N., 2020. A study on using image based machine learning methods to develop the surrogate models of stamp forming simulations.

[46] Kingma, D. P., and Ba, J., 2014. "Adam: A method for stochastic optimization". arXiv preprint arXiv:1412.6980.

[47] Vaswani, A., Shazeer, N., Parmar, N., Uszkoreit, J., Jones, L., Gomez, A. N., Kaiser, L., and Polosukhin, I., 2017. "Attention is all you need.". Neural Information Processing Systems.

[48] Yang, F., Yang, H., Fu, J., Lu, H., and Guo, B., 2020. "Learning texture transformer network for image superresolution.". 2020 IEEE/CVF Conference on Computer Vision and Pattern Recognition (CVPR), Computer Vision and Pattern Recognition (CVPR), 2020 IEEE/CVF Conference on, CVPR, pp. 5790 - 5799.

[49] Kasem, H., Hung, K., and Jiang, J., 2019. "Spatial transformer generative adversarial network for robust image super-resolution.”. IEEE Access, Access, IEEE, 7, pp. $182993-183009$. 